\title{
Fatty acid composition of platelet phospholipids in non-insulin-dependent diabetics randomized for dietary advice
}

\author{
BY I. LOPEZ-ESPINOZA, ${ }^{1}$ J. HOWARD-WILLIAMS, ${ }^{2}$ J. I. MANN, ${ }^{3}$ \\ R. D. CARTER ${ }^{3}$ AND T. D. R. HOCKADA Y ${ }^{1}$ \\ ${ }^{1}$ Sheikh Rashid Diabetes Unit, Radcliffe Infirmary, Oxford OX2 $6 \mathrm{HE}$ \\ ${ }^{2}$ The Eye Hospital, Walton Street, Oxford OX2 $6 \mathrm{HE}$ \\ ${ }^{3}$ Diabetes Research Laboratories, Radcliffe Infirmary, Oxford $O X 26 H E$
}

(Received 19 August 1983 - Accepted 26 January 1984)

1. Two groups of patients with non-insulin-dependent diabetes mellitus (NIDDM), with dietary advice randomized between a low-carbohydrate (LC) diet and a modified-fat (MF) diet, were followed to determine the effect of diet on phospholipid fatty acid composition of platelets and on development of retinopathy in the 7 years following diagnosis. There was a tendency for retinopathy to occur more frequently in those randomized to the LC diet. This difference was not statistically significant, and fatty acid composition of platelets did not differ significantly in those with and without retinopathy.

2. Linoleic acid values from platelet phospholipid fatty acids were significantiy higher in NIDDM on an MF diet compared with an LC diet.

3. There was no difference between the two dietary subgroups with respect to platelet arachidonic acid, but this was lower in the whole diabetic population when compared with non-diabetics.

4. The arachidonic acid values correlated with neither glycosylated haemoglobin nor mean glycaemia.

5. Significant correlation between the fatty acid values for platelets and plasma cholesterol esters was found only for $16: 0$.

The composition of platelet phospholipids can be affected by diet (Nordoy \& Rodset, 1971). After a diet rich in linoleic acid the platelet linoleic acid, but not arachidonic acid, increased. In a study of Eskimos in Greenland, a high consumption of $\omega 3$ polyunsaturated fatty acids increased their proportion among the platelet fatty acids (Dyerberg \& Bang, 1979). In humans, changes in the fatty acids of platelet phospholipids have been associated with changes in platelet function in vitro (Siess et al. 1980). For instance, decreases in platelet aggregation have been observed after dietary supplementation with linoleic acid, and increases after arachidonic acid (Fleischman et al. 1975; Seyberth et al. 1975). Such altered platelet function could depend on reduced formation of thromboxane A 2 as seen in animals given linoleic acid (Mathias et al. 1978) and such a reduction would lessen the tendency to aggregation of platelets (Kloeze, 1967). Houtsmuller et al. (1979) reported that a linoleic acid-rich diet, administered over several years, inhibited the development of microangiopathic retinopathy in diabetic subjects.

We therefore examined the effect on the phospholipid fatty acid composition of platelets in non-insulin-dependent diabetes mellitus (NIDDM) patients with dietary advice randomized between a low-carbohydrate (LC) and a modified-fat (MF) diet. We also wished to determine whether there was any association between the platelet phospholipid values and the development of retinopathy in the preceding 7 years.

\section{SUBJECTS AND METHODS}

Patients with NIDDM ( $n$ 250) were recruited at diagnosis 7-9 years ago to a prospective study and randomized to advice on either a classic low-carbohydrate (LC) diet with $40 \%$ of energy from carbohydrate or a modified-fat (MF) diet with the following main features: (a) restriction of energy from fat to $30 \%$ of total, (b) weekly cholesterol content restricted 
Table 1. The average daily fatty acid composition $(g)$ of low-carbohydrate $(L C)$ and modified-fat $(M F)$ advised diets

(Advice as to energy content was determined by percentage ideal body-weight; the average was $5858 \mathrm{~kJ}$ ( $1400 \mathrm{kcal})$ for the analysed diets)

\begin{tabular}{lrr}
\hline \hline & LC diet & MF diet \\
\hline Total saturated fatty acids & $31 \cdot 7$ & $16 \cdot 7$ \\
$16: 0$ & $16 \cdot 3$ & $8 \cdot 1$ \\
Total monosaturated fatty acids & $29 \cdot 0$ & $14 \cdot 7$ \\
$18: 2$ & 4.9 & $19 \cdot 3$ \\
$18: 3$ & $0 \cdot 6$ & $0 \cdot 7$ \\
$20: 4$ & $1 \cdot 9$ & $0 \cdot 1$ \\
Polyunsaturated : saturated & 0.1 & $1 \cdot 1$ \\
\hline \hline
\end{tabular}

to $2 \mathrm{~g}$, (c) ratio, polyunsaturated: other fatty acids increased to at least 0.9 (Hockaday $e t$ al. 1978). Typical fatty acid compositions of the two advised diets, calculated from McCance and Widdowson's food tables (Paul \& Southgate, 1978), are listed in Table 1. The results are based on dietary interview with six patients at the time of the ophthalmic review.

From this population we measured the platelet phospholipids in fifty-nine diabetics (NIDDM) chosen randomly from the 150 who attended an ophthalmic review 7 years after diagnosis. There were thirty-four men and twenty-five women. Twenty-five were treated by diet alone, twenty-five also took hypoglycaemic sulphonylureas and nine were on insulin. All of them had been reviewed before and 1,2 and 5 years after the start of treatment, and their metabolic-assessment tests included fasting glucose and total cholesterol plasma concentrations. Details of the patients are given in Table 2. Twenty-nine non-diabetic volunteers, taking their usual diet, were studied as controls for the platelet phospholipid values. Their mean age was 42 (SE 10) years and they included twenty-four men and five women.

The presence and extent of retinopathy was determined by fundoscopy and retinal photography after dilating the pupils (Howard-Williams et al. 1984).

Blood was taken using minimal venous occlusion. A sterile 19-gauge needle was introduced into an antecubital vein, and blood was allowed to flow freely through a short length of attached polyethylene tubing into a plastic tube containing trisodium citrate solution $(38 \mathrm{~g} / \mathrm{l})$ for determination of platelet membrane fatty acids by thin-layer (TLC) and gas-liquid chromatography, using a modification of the method of Moore et al. (1977). Once a blood:citrate value of 9:1 was achieved, the mixture was centrifuged at $180 \mathrm{~g}$ for $10 \mathrm{~min}$. Platelet-rich plasma was drawn off with a plastic pipette and the platelets were washed twice in saline $(9 \mathrm{~g}$ sodium chloride/1), with centrifugation at $500 \mathrm{~g}$ after each washing to obtain a platelet pellet. Lipids were extracted by rotary mixing with a chloroform-methanol solvent $(2: 1, \mathrm{v} / \mathrm{v})$ for $10 \mathrm{~min}$ and the phases separated by centrifugation at $350 \mathrm{~g}$ for $5 \mathrm{~min}$. The lower organic phase was evaporated to dryness under a stream of air at $50^{\circ}$. The residue was dissolved in $100 \mu 1$ chloroform and applied separately to aluminium-backed plates of silica gel $60 \mathrm{G}$ (E. Merck, Darmstadt, Germany) which had been activated previously at $100^{\circ}$ for $2 \mathrm{~min}$. TLC plates were developed in hexane-diethyl ether-glacial acetic acid (85:15:1, by vol.) until they had run for a distance of $100 \mathrm{~mm}$, and solvent evaporated at room temperature. The plates were immersed in a tank of iodine vapour for $20 \mathrm{~s}$ to visualize the spots, the phospholipids were scraped off into a clean tube and then hydrolysed by incubation with $1.0 \mathrm{ml}$ freshly prepared $1 \mathrm{M}$-sodium hydroxide in methanol $(950 \mathrm{ml} / 1)$ at $37^{\circ}$ for $20 \mathrm{~min}$. The mixture was acidified by addition of $2.0 \mathrm{ml}$ 
Table 2. Description of patients included in the study

(Values are means and standard deviations)

\begin{tabular}{|c|c|c|c|c|c|}
\hline & \multicolumn{2}{|c|}{ LC diet $(n 25)$} & \multicolumn{2}{|c|}{ MF diet $(n 34)$} & \multirow{2}{*}{$\begin{array}{c}\text { Statistical } \\
\text { significance } \\
\text { of difference: } P\end{array}$} \\
\hline & Mean & SD & Mean & SD & \\
\hline Age (years) & $56 \cdot 0$ & $8 \cdot 5$ & $56 \cdot 7$ & 10.2 & \\
\hline Height (m) & 1.66 & 0.07 & 1.66 & 0.12 & \\
\hline Wt (kg) & $74 \cdot 4$ & $9 \cdot 4$ & $81 \cdot 1$ & 13.9 & $<0.01$ \\
\hline BMI* $^{*}$ & 28.7 & $3 \cdot 3$ & 31.9 & 5.4 & $<0.01$ \\
\hline $\mathrm{BMI}_{\mathrm{O}} \dagger$ & $28 \cdot 0$ & $3 \cdot 3$ & 30.9 & $5 \cdot 6$ & $<0.01$ \\
\hline $\mathrm{BMI}_{\max } \ddagger$ & 31.6 & $2 \cdot 9$ & $35 \cdot 5$ & $5 \cdot 7$ & $<0.0025$ \\
\hline$\triangle \mathrm{BMI}_{\mathrm{O}} \S$ & 0.7 & 1.8 & 1.0 & $2 \cdot 8$ & \\
\hline Duration of diabetes (years) & $7 \cdot 0$ & $1 \cdot 0$ & 7.0 & 0.8 & \\
\hline Medication & \multicolumn{2}{|c|}{ (\% group) } & \multicolumn{2}{|c|}{ (\% group) } & \\
\hline Diet alone & \multirow{2}{*}{\multicolumn{2}{|c|}{$\begin{array}{l}44 \\
44\end{array}$}} & \multicolumn{2}{|c|}{41} & \\
\hline Diet + sulphonylureas & & & \multirow{2}{*}{\multicolumn{2}{|c|}{$\begin{array}{l}41 \\
18\end{array}$}} & \\
\hline Diet + insulin & \multicolumn{2}{|c|}{12} & & & \\
\hline \multicolumn{6}{|l|}{ Retinopathy } \\
\hline None & \multicolumn{2}{|c|}{52} & \multicolumn{2}{|c|}{59} & \\
\hline Background & \multicolumn{2}{|c|}{48} & \multicolumn{2}{|c|}{41} & \\
\hline $\operatorname{Sex}\left(0^{\pi}: P\right)$ & \multicolumn{2}{|c|}{$18: 7$} & \multicolumn{2}{|c|}{$16: 8$} & \\
\hline
\end{tabular}

LC, low-carbohydrate; MF, modified fat.

* BMI, body mass index (weight $(\mathrm{kg}) /$ height $(\mathrm{m})^{2}$ ) at diagnosis.

$\dagger$ At ophthalmic review.

$\ddagger$ Calculated from maximum remembered weight.

$\S$ Change from time of diagnosis to ophthalmic review.

$1 \mathrm{M}$-hydrochloric acid. The fatty acids were extracted twice with $2.0 \mathrm{ml}$ diethyl ether and then methylated by addition of $0.2 \mathrm{ml}$ methanol and diazomethane in diethyl ether until a yellow colour was retained. After standing at room temperature for $10 \mathrm{~min}$, the extract was evaporated to dryness under a stream of air at $50^{\circ}$. The methylated portions were analysed by gas-liquid chromatography in a Pye Unicam model 104 gas chromatograph (Pye Unicam, Cambridge), using a $2 \mathrm{~m}$ glass column with $10 \%$ diethylene glycol succinate on a ' gas chrom Q 80-100' mesh support. Peak areas were calculated by triangulation and each fatty acid was expressed as a percentage of the total fatty acids. No special precaution was taken to protect platelet lipids from auto-oxidation. However, analysis of the samples was started almost immediately after the blood had been taken, which removes many of the problems. Furthermore, in our laboratory we have produced very similar results (far from statistically significantly different) when the method described here has been compared with a procedure including the addition of gas antioxidant, the drying of lipids under nitrogen rather than air and avoiding the use of $I_{2}$ to visualize spots on the TLC plate. Samples were taken for glucose analysis by a glucose oxidase (EC 1.1.3.4) method in a Pye Unicam $\mathrm{ACl}$ autoanalyser.

All total cholesterol measurements were performed on a Technicon AutoAnalyzer II according to the Lieberman-Burchard reaction (Huang et al. 1961). The fatty acids of cholesterol esters were determined using the Moore et al. (1977) method. Cholesterol in the lipoprotein subfractions was assayed after precipitation with heparin, manganese chloride (Burstein et al. 1970) and sodium dodecyl sulphate (Ononongba \& Lewis, 1976). Glycosylated haemoglobin ( $\mathrm{HbA}_{1 \mathrm{c}}$ ) was measured by isoelectric focusing (Jeppsson \& Berglund, 1972).

Statistical analysis included mean and standard deviation, with Student's $t$ test to measure the significance of differences, Spearman's rank, and Pearson partial correlation coefficients to assess correlation. 
Table 3. The fatty acid composition of the platelet phospholipids $(\%$ total phospholipid fatty acids) in non-insulin-dependent diabetes mellitus (NIDDM) patients and in non-diabetics (ND)

(Values are means and standard deviations)

\begin{tabular}{|c|c|c|c|c|c|c|c|c|c|c|c|}
\hline \multirow[b]{3}{*}{ Fatty acid } & & & \multicolumn{6}{|c|}{ NIDDM } & \multirow{2}{*}{\multicolumn{3}{|c|}{$\begin{array}{c}\text { Statistical } \\
\text { significance of } \\
\text { comparison between } \\
\text { groups }\end{array}$}} \\
\hline & \multicolumn{2}{|c|}{$\mathrm{ND}(\mathrm{A} ; n 29)$} & \multicolumn{2}{|c|}{ Total (B; $n$ 59) } & \multicolumn{2}{|c|}{ LC diet $(C ; n$ 25) } & \multicolumn{2}{|c|}{$\mathrm{MF} \operatorname{diet}(\mathrm{D} ; n$ 34) } & & & \\
\hline & Mean & SD & Mean & SD & Mean & SD & Mean & SD & A v. B & A v. C & A v. D \\
\hline $16: 0$ & $18 \cdot 5$ & $3 \cdot 3$ & $16 \cdot 3$ & $4 \cdot 0$ & $15 \cdot 5$ & $4 \cdot 0$ & $16 \cdot 6$ & $4 \cdot 1$ & * & $*$ & * \\
\hline $18: 0$ & $16 \cdot 4$ & $2 \cdot 1$ & $15 \cdot 1$ & $3 \cdot 0$ & $15 \cdot 2$ & $3 \cdot 3$ & $15 \cdot 1$ & 2.9 & * & NS & $*$ \\
\hline $18: 1$ & $15 \cdot 8$ & $2 \cdot 8$ & $15 \cdot 1$ & $2 \cdot 9$ & 15.4 & $3 \cdot 4$ & 14.8 & $2 \cdot 5$ & NS & NS & NS \\
\hline $18: 2066$ & $10 \cdot 2$ & $2 \cdot 9$ & $12 \cdot 4$ & $3 \cdot 1$ & $11 \cdot 3$ & $2 \cdot 7$ & $13 \cdot 1$ & $3 \cdot 1$ & $* * *$ & NS & $* * *$ \\
\hline $20: 3 \omega 9$ & 1.7 & $1 \cdot 5$ & 1.8 & 0.8 & 1.9 & $1 \cdot 1$ & 1.6 & 0.5 & NS & NS & NS \\
\hline $20: 4 \omega 6$ & $22 \cdot 3$ & $3 \cdot 4$ & $19 \cdot 5$ & 3.8 & $19 \cdot 4$ & $3 \cdot 8$ & $19 \cdot 7$ & 4.0 & $*$ & $*$ & $*$ \\
\hline $20: 5 \omega 3$ & $1 \cdot 2$ & 0.4 & 1.4 & 0.8 & 1.4 & 0.9 & $1 \cdot 3$ & 0.7 & NS & NS & NS \\
\hline
\end{tabular}

LC, low-carbohydrate; MF, modified fat; NS, not significant.

* $P<0.025, * * * P<0.001$.

\section{RESUL.TS}

There was no significant difference in mean age between the LC and MF groups at the beginning of the study (Table 2). The body mass index (BMI; weight/height ${ }^{2}$ ) was higher in the MF group at the beginning of the study and at the ophthalmic review than the LC group; there was no difference in BMI change from time of diagnosis to ophthalmic review in both groups (Table 2). Retinopathy was detected after approximately 7 years in $48 \%$ of the LC group and $41 \%$ of the MF group.

The mean fasting total plasma cholesterol $(\mathrm{mmol} / \mathrm{l})$ for the first, third and fifth years of the study was significantly different between the two groups: LC, mean 5.60 (SD 1.03); MF, mean 5.07 (SD 0.84) $(P<0.05)$. However, at the ophthalmic review the random total cholesterol value (mmol/1) did not differ: LC, mean 4.87 (SD 0.79); MF, mean 4.94 (SD 0.82). The plasma fatty acid composition of the cholesterol esters ( $\%$ total fatty acids) was

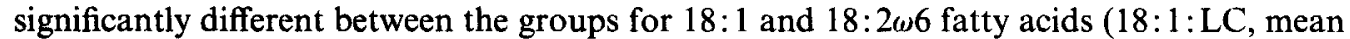

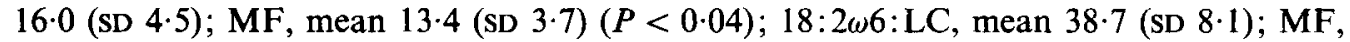
mean 44.5 (SD 9.2) $(P<0.01)$. There was no other statistically significant difference.

The fatty acid values from platelet phospholipids are presented only for those fatty acids present in appreciable amounts $(>10 \%)$ or when statistically significant differences or correlations emerged (Tables 3-5).

The percentage of linoleic acid $(18: 2 \omega 6)$ from platelet phospholipids was significantly higher in the MF than the LC group (MF, mean 13.1 (SD 3.1); LC, mean 11.3 (SD 2.7) $(P<0.02))$.

The percentages of 16:0,18:0 and 20:4 6 platelet phospholipids were significantly lower in the diabetic group as a whole when compared with the non-diabetics (Table 3 ). This also held for either dietary group separately, except for 18:0 in the LC-diabetic group. However, while the percentage of $18: 2 \omega 6$ was higher in all diabetics when compared with normals,

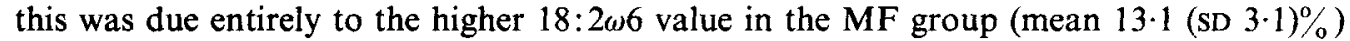
and was not so for the LC group. The $\mathrm{HbA}_{1 \mathrm{c}}(\%)$ was very similar in the two groups: LC, mean $8 \cdot 1$ (SD 2.2); MF, mean $7 \cdot 8$ (SD 2.1).

When the diabetics were divided into those with and without retinopathy, irrespective 
Table 4. Composition of fatty acids in the platelet phospholipids $(\%$ total phospholipid fatty acids)

(Values are means and standard deviations)

\begin{tabular}{|c|c|c|c|c|}
\hline \multirow[b]{2}{*}{ Fatty acid } & \multicolumn{2}{|c|}{ Retinopaths ( $n$ 26) } & \multicolumn{2}{|c|}{ Non-retinopaths ( $n$ 32) } \\
\hline & Mean & SD & Mean & SD \\
\hline $16: 0$ & 16.9 & $3 \cdot 5$ & $15 \cdot 3$ & $4 \cdot 3$ \\
\hline 18:0 & 15.5 & $2 \cdot 8$ & 15.0 & $3 \cdot 2$ \\
\hline $18: 1$ & 15.4 & $2 \cdot 6$ & 14.5 & $2 \cdot 9$ \\
\hline $18: 2 \omega 6$ & 12.7 & 2.7 & $12 \cdot 0$ & $3 \cdot 4$ \\
\hline $20: 1 \omega 9$ & $0 \cdot 1$ & 0.1 & 0.2 & $0 \cdot 3$ \\
\hline $20: 3 \omega 9$ & 1.8 & $1 \cdot 1$ & 1.7 & 0.5 \\
\hline $20: 4 \omega 6$ & 19.5 & $3 \cdot 1$ & 19.9 & $4 \cdot 1$ \\
\hline $20: 5 \omega 3$ & $1 \cdot 3$ & 0.6 & 1.4 & 0.9 \\
\hline
\end{tabular}

Table 5. Fatty acids from platelet phospholipids in patients with non-insulin-dependent diabetes mellitus with and without retinopathy, on low-carbohydrate $(L C)$ and modified-fat $(M F)$ diets (Values are means and standard deviations)

\begin{tabular}{|c|c|c|c|c|c|c|c|c|c|c|c|c|}
\hline \multirow{3}{*}{$\begin{array}{l}\text { Diet... } \\
\text { Fatty acid }\end{array}$} & \multicolumn{4}{|c|}{ Retinopaths } & \multicolumn{4}{|c|}{ Non-retinopaths } & \multirow{2}{*}{\multicolumn{4}{|c|}{$\begin{array}{c}\text { Statistical significance of } \\
\text { comparison between groups }\end{array}$}} \\
\hline & \multicolumn{2}{|c|}{$\mathrm{LC}(\mathrm{A} ; n 12)$} & \multicolumn{2}{|c|}{$\mathrm{MF}(\mathrm{B} ; n$ 14) } & \multicolumn{2}{|c|}{$\mathrm{LC}(\mathrm{C} ; n 12)$} & \multicolumn{2}{|c|}{$\mathrm{MF}(\mathrm{D} ; n 20)$} & & & & \\
\hline & Mean & SD & Mean & SD & Mean & $\mathrm{SD}$ & Mean & SD & A v. B & A v. C & $\mathrm{C} v . \mathrm{D}$ & $\mathrm{B} v . \mathrm{D}$ \\
\hline $16: 0$ & $15 \cdot 7$ & 3.9 & $18 \cdot 0$ & $2 \cdot 9$ & $16 \cdot 4$ & $2 \cdot 6$ & $15 \cdot 5$ & 4.4 & * & NS & NS & * \\
\hline $18: 0$ & $16 \cdot 3$ & $2 \cdot 8$ & $14 \cdot 8$ & $2 \cdot 9$ & $14 \cdot 7$ & $3 \cdot 3$ & 16.0 & 1.9 & NS $^{\circ}$ & NS & NS & NS \\
\hline $18: 1$ & $15 \cdot 5$ & 2.9 & 15.0 & $2 \cdot 4$ & $15 \cdot 7$ & $3 \cdot 2$ & $14 \cdot 4$ & $1 \cdot 6$ & NS & NS & NS & NS \\
\hline $18: 2 \omega 6$ & $12 \cdot 3$ & $2 \cdot 7$ & $13 \cdot 3$ & $2 \cdot 8$ & $10 \cdot 9$ & $2 \cdot 7$ & 13.4 & $2 \cdot 7$ & NS & NS & $* *$ & NS \\
\hline $20: 3 \omega 9$ & $2 \cdot 1$ & $1 \cdot 5$ & 1.6 & 0.5 & $1 \cdot 7$ & 0.4 & 1.8 & 0.5 & NS & NS & NS & NS \\
\hline $20: 4 \omega 6$ & $19 \cdot 1$ & 3.5 & $19 \cdot 6$ & $2 \cdot 8$ & $20 \cdot 0$ & 3.4 & $20 \cdot 3$ & $4 \cdot 0$ & NS & NS & NS & NS \\
\hline $20: 5 \omega 3$ & $1 \cdot 1$ & 0.6 & $1 \cdot 5$ & 0.7 & $1 \cdot 8$ & $1 \cdot 2$ & $1 \cdot 2$ & 0.8 & NS & $\bullet$ & $*$ & NS \\
\hline
\end{tabular}

NS, not significant.

${ }^{*} P<0.05,{ }^{* *} P<0.01$

of dietary advice, there was no difference in the platelet phospholipids between the groups (Table 4). The only difference in the fatty acid composition of plasma cholesterol esters was that the 18:0 ester (\%) was significatly higher in the retinopathy group (retinopaths, mean 1.1 (SD 0.9); non-retinopaths, mean 0.67 (SD 0.7) $(P<0.05)$ ). However, when the dietary groups were considered separately, significant differences appeared among the phospholipid values. In retinopaths, 16:0 (\%) was lower in LC, mean 15.7 (SD 3.9), than MF, mean 18.0 (SD 2.9) $(P<0.05)$. The levels of 18:2 $26(P<0.01)$ and 20:5 $(P<0.05)$ were significantly different on comparing $\mathrm{LC}$ and $\mathrm{MF}$ in the non-retinopaths (Table 5).

The mean level of blood glucose at $0,1,3$ and 5 years correlated inversely and significantly (but weakly) with the percentage of three fatty acids in the platelet phospholipids for all

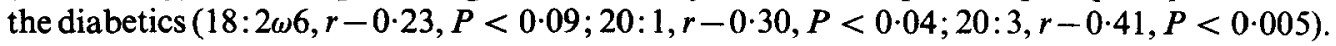
20:1 Also correlated significantly with 18:2 $(r 0.53, P<0.001)$ but neither correlated with $20: 3$. On partial correlation analysis, there was no significant correlation between glucose and $18: 2$ or $20: 1$ on allowing for interactions with $20: 3$, but $20: 3$ strongly and persistently

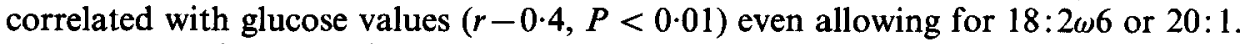

On comparing the platelet fatty acids with the plasma cholesterol ester values, a 
significant relationship was found between the plasma cholesterol esters and platelet fatty acids only for $16: 0(r 0.31, P<0.008)$.

\section{DISCUSSION}

Diets rich in polyunsaturated fats have been used to reduce cholesterol in diabetics (Stone

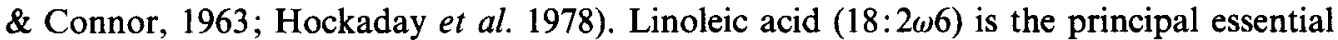
fatty acid present in many of these diets. It is a common precursor for dihomo- $\gamma$-linoleic $(20: 3)$ and arachidonic $(20: 4 \omega 6)$ fatty acids.

The linoleic acid fraction $(18: 2 \omega 6)$ from platelet phospholipid fatty acids was significantly higher in NIDDM patients on an MF diet compared with either the non-diabetic volunteers (on their normal diet) or the NIDDM on an LC diet. This accords with previous reports which have shown dietary influences on platelet fatty acid composition (Nordoy \& Rodset, 1971; Dyerberg \& Bang, 1979; Siess et al. 1980).

The percentage of platelet arachidonic acid seems to vary in different populations studied (Dyerberg \& Bang, 1979; Siess et al. 1980; Sanders et al. 1981). However, in our population the diabetic group as a whole had a significantly lower percentage of arachidonic acid than the normal group. There was no difference, however, between the two dietary subgroups. This confirms the findings of Jones et al. (1983), working with insulin-treated diabetics, and suggests that the percentage of arachidonic acid from platelet phospholipids is independent of dietary intake of linoleic acid. This agrees with the observation of Nordoy \& Rodset (1971) who found non-diabetic subjects on a soya-bean oil diet to have increased linoleic platelet phospholipid-fatty acid without change in arachidonic acid.

Arachidonic acid is the precursor of the prostaglandins containing two double bonds, PGE 2, PGD2, PGI 2 and thromboxane A2. This last substance is a potent vascoconstrictor and is the most potent platelet-agreggating agent known (Gryglewski, 1980). Thromboxane A 2 breaks down spontaneously into thromboxane B2, a stable compound devoid of biological activity, which has been shown to be raised in diabetics (Ylikorkala et al. 1981). Platelets from the blood of diabetic subjects show an increased susceptibility to aggregate in vitro (Sagel et al. 1975) and this property could be implicated in the pathogenesis of diabetic angiopathy. We suggest that the decrease in arachidonic acid found in this and other studies could be associated, via its increased conversion to thromoboxane A2, with the increased aggregation of platelets in diabetics. The positive correlation between platelet phospholipid-linoleic acid and mean glycaemic control is reminiscent of that previously reported for $\mathrm{HbA}_{1 \mathrm{c}}$ and linoleic acid in a combined group of diabetics and non-diabetics (Jones et al. 1983), but there the cornelation did not hold for the diabetics alone (all insulin-treated). In contrast to this previous study, we found no correlation between arachidonic acid and either $\mathrm{HbA}_{1 \mathrm{c}}$ or mean glycaemia. The correlations between glycaemia and 20:1 and 20:3 fatty acids should not be emphasized because of the low absolute levels of these two fatty acids.

The lack of difference in total plasma cholesterol between the LC and MF groups here, in contrast to that 1, 3 and 5 years after start of treatment, may be due to a true loss of the dietary effect with time, but could be explained by the difference between post-prandial bloods here and fasting bloods earlier in the study. Significant diurnal variation in plasma cholesterol concentration has been reported (Simpson et al. 1980) and it is larger in diabetics than normal subjects.

A higher intake of linoleic acid may be necessary for this fatty acid to exceed $50 \%$ of the cholesterol ester-fatty acids, and so provide circumstances more suitable to test the general applicability of the striking results of Houtsmuller et al. (1979). 
The authors are grateful to Dr D. Jones, Dr R. Yajnik and Mr R. Smith for their advice and help; to Mrs P. Booth for her skilled nursing assistance, and Mr R. Jelfs for technical assistance. Miss P. Patel assisted with the dietary instruction of patients on the two diets. Statistical analyses were performed by Miss V. Beckett. This study was supported by the Simon Broome Heart Research Trust and the Oxford Diabetes Trust funding of the Sheikh Rashid Diabetes Unit.

\section{REFERENCES}

Burstein, M., Scholnick, H. R. \& Morfin, R. (1970). Journal of Lipid Research 11, 583-595.

Dyerberg, J. \& Bang, H. O. (1979). Lancet i, 433-435.

Fleischman, A. I., Bierembaum, M. L., Justice, D., Stier, A., Sullivan, A. \& Fleischman, M. (1975). American Journal of Clinical Nutrition 28, 601-605.

Gryglewski, R. J. (1980). CRC Critical Reviews in Biochemistry 7, 291-338.

Hockaday, T. D. R., Hockaday, J. M., Mann, J. I. \& Turner, R. C. (1978). British Journal of Nutrition 39, $357-362$.

Houtsmuller, A. J., Zahn, K. J. \& Henker, H. E. (1979). Documenta Ophthalmologica 48, 363-371.

Howard-Williams, J., Patel, P., Jelfs, R., Carter, R. D., Awdry, P., Bron, A., Mann, J. I. \& Hockaday, T. D. R. (1984). British Journal of Ophthalmology (In the Press).

Huang, T. C., Chen, C. P., Wefler, V. \& Raftery, A. (1961). Analytical Chemistry 33, 1405-1407.

Jeppson, J. O. \& Berglund, S. (1972). Clinica Chimica Acta 40, 153-158.

Jones, D. B., Carter, R. D., Haitas, B. \& Mann, J. I. (1983). British Medical Journal 286, 173-175.

Kloeze, J. (1967). In Prostaglandins: Proceedings of 2nd Nobel Symposium, pp. 241-250. [S. Bergstrom and B. Samuelsson, editors]. New York: Interscience.

Mathias, M. M., Fine, K., Lipinski, B. \& Dupont, J. (1978). Prostaglandins 15, 706.

Moore, R. A., Oppert, S., Eaton, P. \& Mann, J. I. (1977). Clinical Endocrinology 7, 143-149.

Nordoy, A. \& Rodset, M. (1971). Acta Medica Scandinavica 190, $27-34$.

Ononongba, I. C. \& Lewis, B. (1976). Clinica Chimica Acta 71, $397-402$.

Paul, A. A. \& Southgate, D. A. T. (1978). McCance and Widdowson's The Composition of Foods, pp. $293-300$. London: H.M. Stationery Office.

Sagel, J., Colwell, J. A., Crook, L. \& Laimins, M. (1975). Annals of Internal Medicine 82, 733-738.

Sanders, T. A., Vickers, M. \& Haines, A. P. (1981). Clinical Science 61, 317-324.

Seyberth, H. W., Oelz, O., \& Kennedy, T. (1975). Clinical Pharmacology and Therapeutics 18, 521-529.

Siess, W., Scherer, B., Bohlig, B., Roth, P., Kurzmann, I. \& Weker, P. C. (1980). Lancet i, 441-444.

Simpson, R. W., Carter, R. D., Moore, R. A. \& Penfold, W. A. F. (1980). Diabetologia 18, $35-40$.

Stone, D. B. \& Connor, W. E. (1963). Diabetes 12, $127-131$.

Ylikorkala, O., Kaila, J. \& Viinika, L. (1981). British Medical Journal 283, 1148-1150. 